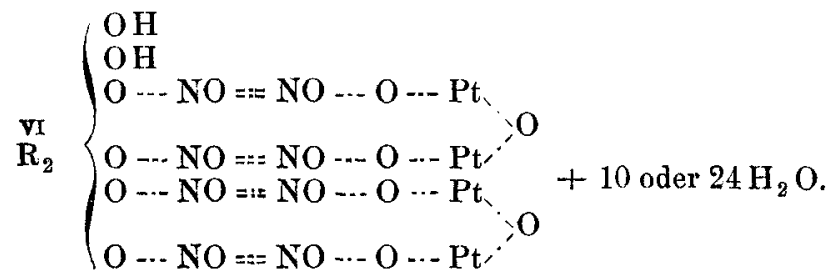

Es ist beachtenswerth, dass schwache Basen vorzugsweise geneigt sind, solche Verbindungen zu bilden. Unter den einwerthigen Metallen ist es nämlich Silber, unter den zweiwerthigen Beryllium und unter den sechswerthigen $\mathbf{R}_{2}$ Aluminium, Chrom, Eisen, Indium, welche entweder nur nach Verdampfen der Lösungen im Wasserbade oder auch unter allen Umständen die erwähnten Condensationsprodukte gebildet haben. Doch habe ich die Bildung solcher rothen Produkte auch in mehreren anderen Fällen beobachtet, besonders wenn die Platonitritlösungen [z. B. von $\mathrm{Am}, \mathrm{Ca}, \mathrm{Hg}, \mathrm{Y}, \mathrm{Er}$ etc.] über Schwefelsäure vollkommen eintrockneten; doch fand diese Zerlegung nur spurenweise statt, und dadurch wurde jede Untersuchung der gebildeten Produkte verhindert.

Upsala, Universitäts-Laboratorium, den 18. November 1876.

464. Albert Atterberg: Ueber einige aus den $\alpha$ - und $\beta$-Dinitronaphtalinen dargestellte Naphtalinderivate.

(Eingegangen am 21. November; verl. in der Sitzung von Hru. Oppenheim.)

Bei der Behandlung des $\alpha$-Dinitronaphtalins mit Phosphorpentachlorid entsteht, wie ich nenlich (d. Ber. IX, 1188) nachgewiesen habe, dasselbe bei $107^{\circ}$ schmelzendes Dichlornaphtalin, das früher von mir aus Nitronaphtalin dargestellt worden ist (d. Ber. IX, 317). Da es durch die neue Darstellungsmethode mir möglich war grössere Mengen dieses Dichlornaphtalins za bereiten, habe ich davon folgende Derivate dargestellt.

Nitro- $\gamma$-dichlornaphtalin, $\mathrm{C}_{10} \mathrm{H}_{5} \mathrm{ClNO}_{2}$, ist von mir schon (d. Ber. IX, 928) beschrieben. Bei der Analyse desselben wurde gefunden $29.17 \mathrm{pCt}$. Chlor und $5.70 \mathrm{pCt}$. Stickstoff (berechnet 29.34 und $5.79 \mathrm{pCt}$.) Es ist das einzige Produkt bei der Nitrirung des $\gamma$-Dichlornaphtalins mit Salpetersäure von 1.4. Durch Phosphorpentachlorid wird es in $\delta$-Trichlornaphtalin übergeführt (d. Ber. IX, 1187). Bej Behandlung desselben mit einer Mischung von Schwefelsäure und Salpetersäure entsteht daraus

Dinitro- $\gamma$-dichlornaphtalin, $\mathrm{C}_{10} \mathrm{H}_{4} \mathrm{Cl}_{2} \cdot 2 \mathrm{NO}_{2}$. Diese Verbindung bildet hellgelbe, spröde, prismatische Nadeln, die soga 
in Eisessig sehr schwer löslich sind. Einmal aus Eisessig krystallisirt, ist sie schon vollkommen rein und zeigt den constanten Schmelzpunkt $246^{\circ}$. Es wird nicht ron concentrirtester Kalilauge angegriffen. Die Analyse davon ergab $24.45 \mathrm{pCt}$. Chlor und $10.41 \mathrm{pCt}$. Stickstoff (berechnet 24.74 and $9.76 \mathrm{pCt}$.).

Um die Stellung der Nitrogruppe der vorigen Mononitroverbindung näher zu bestimmen, habe ich versucht durch längere Behandlung mit Zinn und Salzsäure die beiden Chloratome darin durch Wasserstoff zu ersetzen, um so ein Naphtylamin zu erhalten. Die Reduction konnte aber nicht weiter geführt werden als bis zur Bildung eines Monochlornaphtylamins, das als schwerlösliches Zinndoppelsalz sich abschied. Da ich inzwischen durch eine Synthese anderer Art gewiss wurde, dass die Nitrogruppe des Nitro- $\gamma$-dichlornaphtalins die $\alpha$-Stellung besitzt, habe ich nicht versucht, die Reduction vollständiger zu machen. Von der entstandenen Aminbase habe ich jedoch folgende Verbindungen untersucht.

Das Zinndoppelsalz des Chlornaphtylamins,

$$
\mathrm{C}_{10} \mathrm{H}_{6} \mathrm{Cl} . \mathrm{NH}_{2} . \mathrm{HCl}+\mathrm{SnCl}_{2}
$$

krystallisirt in grossen, schönen Krystallblättern, die in Wasser ziemlich schwer löslich sind. Die Analyse des Salzes ergab 30.69 pCt. Zinn und 35.20 pCt. Chlor (berechnet 29.28 und 35.23 pCt.).

Chlornaphtylamin-hydrochlorat, $\mathrm{C}_{10} \mathrm{H}_{6} \mathrm{Cl} . \mathrm{H}_{2} \mathrm{~N} . \mathrm{HCl}$ $+\mathrm{H}_{2} \mathrm{O}$, wird erhalten, wenn aus der Lösung des vorigen Salzes das Zinn durch Schwefelwasserstoff abgeschieden wird. Es krystallisirt aus concentrirter Auflösung in langen, schönen Prismen oder bei zu schneller Krystallisation in flockigen Massen. Das Salz verliert bei $100^{\circ}$ sein Krystallwasser und verflüchtigt sich langsam. Aus der Auflösung des Salzes konnte mit Silbernitrat 15.43 pCt. Chlor ansgefällt werden (berechnet 15.30). Das bei $100^{\circ}$ getrocknete Salz ergab durch Glühen mit Kalk einen Chlorgehalt von 32.84 pCt. (berechnet $33.17 \mathrm{pCt}$.).

Saures Chlornaphtyla min-sulfat, $\mathrm{C}_{10} \mathrm{H}_{6} \mathrm{ClH}_{2} \mathrm{~N}_{2} \mathrm{H}_{2} \mathrm{SO}_{4}$, wird erbalten, wenn man eine Auflösung des Hydrochlorats mit einem Ueberschuss von Schwefelsäure versetzt und die Flüssigkeit darauf durch Verdampfen sehr eingeengt wird. Das Salz krystallisirt in Bündeln von länglichen Blättern, die in Wasser langsam sich lösen. Von kochendem Wasser wird es zum Theil zersetzt, indem die freie Aminbase sich in geschmolzenem Zustande abscheidet. Dasselbe Verhalten findet sich in noch höherem Grade bei dem chlorwasserstoffsaurem Salze wieder. Eine Schwefelbestimmung des Salzes ergab 11.57 pCt. Schwefel (berechnet $11.62 \mathrm{pCt}$.).

Chlornaphtylamin, $\mathrm{C}_{10} \mathrm{H}_{6} \mathrm{Cl} . \mathrm{H}_{2} \mathrm{~N}$, in freiem Zastande durch Fällung des Hydrochlorats mit Ammon dargestellt," scheidet sich ab in weissen, flockigen Massen, die getrocknet bei $93-94^{\circ}$ schmelzen. 
Das Amin ist eine sehwache Base, wie das Verhalten der Salze zu kochendem Wasser deutlich zeigt. Es hat keinen an $\alpha$-Napbtylamin erinnernden Geruch. Die Salze geben mit Eisenchlorid graugrüne Färbung der Flüssigkeit and dann Fällung. Beim Glühen des Zinndoppelsalzes mit Kalk wurden einige Tropfen eines Destillats bekommen, das den Geruch und die Eisenchlorid-Reaction des $\alpha$-Naphtylamins zeigte, was dafür spricht, dass die Amidogruppe des Chlornaphtylamins dieselbe Stellung besitzt, wie die Amidogruppe des $\alpha$-Naphtylamins. Einen mehr entscheidenden Beweis dafür werde icb später anführen.

Das $\beta$-Dinitronaphtalin lieferte, wie ich früher (d. B. IX, 1188) gefunden habe, mit Phosphorpentachlorid statt eines Dichlornaphtalins hauptsächlich nur ein Trichlornaphtalin. In den beim Umkrystallisiren dieses Trichlornaphtalins entstehenden Mutterlaugen erwartete ich das gesuchte Dichlornaphtalin vielleicht auffinden za können und habe ich darum dieselbe Reaction noch einmal in grösserem Maassstabe ausgeführt. Ungeachtet wiederbolten Umkrystallisirens und genauer Sammlung und Untersuchung aller Mutterlaugen konnte ich nicht andere Krystallisationen als die das Trichlornaphtalin kennzeichnende bekommen. Erst wenn die letzten Matterlaugen längere Zeit in der Ruhe gestanden hatten, fand ich darin ziemlich grosse, wohlausgebildete, rhomboëdrische, in den langen Nadeln des Trichlornaphtalins eingebettete Krystalle angeschossen. Diese wurden herausgelesen und einmal aus Alkohol umkrystallisirt. Sie zeigten dann den Schmelzpunkt $83^{\circ}$. Die Chlorbestimmung ergab einen Chlorgehalt von $35.57 \mathrm{pCt}$. (ber. $36.04 \mathrm{pCt}$.) und die Verbindung war also das gesuchte Dichlornaphtalin. Durch die Krystallform onterseheidet sich die neue Verbindung von den früher bekannten Chlornaphtalinen, und sie mag daher als $\zeta$-Dichlornaphtalin bezeichnet werden. Der gefundene Schmelzpunkt derselben kann jedoch noch nicht als sicher angesehen werden, da bei der unbedeutenden Quantität der erhaltenen Substanz sie nur einmal umkrystallisirt werden konnte.

Ein Versuch wurde ferner gemacht, dasselbe Dichlornaphtalin durch Bebandlung von geschmolzenem $\beta$-Dinitronaphtalin mit Chlorgas zu bereiten. Nachdem das Reactionsprodukt durch Destillation mit überbitzten Wasserdämpfen von unangegriffener Nitroverbindung getrennt worden war, konnte jedoch darin durch fractionirtes Krystallisiren nur dasselbe Trichlornapbtalin nebst wenig über $160^{\circ}$ schmelzendem Tetrachlornaphtalin nachgewiesen werden, aber kein Dichlornaphtalin. Ich habe übrigens aus dem oben beschriebenen mit $\beta$-Dinitronaphtalin vielleicht ähnlich constituirten Chlornaphtylamin ein Dichlornaphtalin herzustellen versucht, aber ohne guten Erfolg.

$\delta$-Trichlornaphtalin, $\mathrm{C}_{10} \mathrm{H}_{5} \mathrm{Cl}_{3}$. Dieses Hauptprodukt der Behandlung des $\beta$-Dinitronaphtalins mit Chlor oder Phosphorpenta- 
chlorid entsteht auch, wie ich früher angeführt habe (d. Ber. IX, 1187), durch Einwirkung des Phosphorpentachlorids auf Nitro- $\gamma$-dichlornaphtalin und $\alpha$-Dinitrochlornaphtalin. Ich habe jetzt dieselbe Verbindung nach derselben Methode auch aus $\beta$-Dinitrochlornaphtalin bekommen und $\mathrm{Hr}$. Widm an hat im hiesigen Universitäts-Laboratorium gleichfalls dicselbe aus einer bei $92^{\circ}$ schmelzenden Mononitroverbindung des $\beta$-Dichlornaphtalins dargestellt.

Das aus so vielen Verbindungen darstellbare Trichlornaphtalin lässt sich jedoch nicht ganz leicht in reinem Zustande darstellen. Aus Dinitroverbindungen bereitet ist es immer mit höheren Chlorverbindungen stark verunreigt, und wenn man solche Präparate umkrystallisirt, bleiben diese Verunreinigungen nicht in den Mutterlaugen, sondern sammeln sich in den (doch wohlausgebildeten) Krystallen und erhöhen den Schmelzpunkt derselben. Solche Produkte kann man darum nor durch wiederholtes, fractionirtes Umkrystallisiren in grösserem Maassstabe reinigen, was sehr lästig ist. Als Beispiel der Schwierigkeit solche Vermischungen zu trennen, will ich anführen, dass ich aus $\beta$-Dinitrochlornaphtalin mit Phosphorpentachlorid ein Produkt erhielt, das ans Alkohol wiederholt umkrystallisirt, schliesslich den constanten Schmelzpunkt $136^{\circ}$ zeigte. Bei zwei Chlorbestimmungen fanden sich aber darin $49.17-49.44 \mathrm{pCt}$. Chlor, was einer Mischung von gleichen Molekülen des Tri- und Tetrachlornaphtalins entspricht (berechnet 49.95 pCt.). Durch Krystallisiren aus Eisessig konnte aber diese Substanz allmälich weiter zerlegt werden.

Am reinsten wird aber das $\delta$-Trichlornaphtalin aus den Mononitroderivaten des $\beta$ - und $\gamma$-Dichlornaphtalin bereitet. Diese erfordern nämlich für ibre Reaction auf Phosphorpentachlorid keine so hohe Temperatur wie die Dinitro-Verbindungen und werden darum nicht so leicht durch höhere Chlornaphtaline verunreinigt. Auch hier sind jedoch mannigfache Umkrystallisationen des Rohprodakts unvermeidlich um schliesslich eine reine Verbindung za erzielen.

Das $\delta$-Trichlornaphtalin zeigt im reinen Zustande den Schmelzpunkt $131^{\circ}$. (Der früher von mir angegebene Schmelzpunkt $129^{\circ}$ muss für das angewandte Thermometer mit $+2^{0}$ corrigirt werden.) Es krystallisirt, rein oder unrein, in sebr langen, weichen, platten Nadeln, die sich in Alkohol und Eisessig bei Erwärmung leicht lösen und beim Abkühlen gut auskrystallisiren. Es wird von Salpetersäure leicht angegriffen. Das Produkt ist aber nur eine ölige Masse, woraus ich keine krystallisirbare Nitroverbindung darzustellen vermochte. Es liess sich jedoch daraus ein gelber Körper abscheiden, der die Lösungsmittel stark färbte und aus Alkohol in tiefgelben, stark glänzenden Nadeln auskrystallisirte. Nach den äusseren Eigenschaften des Körpers und nach einer (mit sehr wenig Substanz vorgenommenen) Chlorbestimmung (gef. $25.0 \mathrm{pCt}$., berechn. $26.1 \mathrm{pCt}$. Chlor) scheint die 
Verbindung als ein Dichlornitronaphtochin aufgefasst werden zu dürfen. Bei der Behandlung mit Chromsäure scheint das Trichlornaphtalin gleichfalls ein Chinon zu geben, aber nur in sehr geringer Menge.

Um die Stellung der Chloratome des $\delta$-Trichlornaphtalins näher zu bestimmen, habe ich dasselbe durch Erhitzen mit Salpetersäure in zugeschmolzenen Röhren in eine Phtalsäure übergeführt. Diese Säure, die ein schön sublimirendes Anhydrid lieferte, zeigte einen Chlorgehalt von $29.45 \mathrm{pCt}$. Sie war also eine Dichlorphtalsäure (berechnet $30.21 \mathrm{pCt}$. Chlor), woraus zu schliessen ist, dass von den Chloratomen des $\delta$-Trichlornaphtalins nur zwei in derselben Hälfte des Naphtalinmoleküls sich befinden.

\section{Albert Atterberg: Ueber die Constitution einiger Naphtalinverbindungen.}

(Eingegangen am 21. November; verl. in der Sitzung v. Hrn. Oppenheim.)

Bei dem Fortgang meiner Untersuchungen über Naphtalinverbindungen habe ich einige Verhältnisse aufgefunden; die zur Beurtheilung der rationellen Constitution verschiedener Naphtalinverbindungen Mittel liefern können. Ich will das hierber Gehörige jetzt kürzlich zusammenstellen.

Das von mir dargestellte, bei $131^{\circ}$ schmelzende 8 -Trichlornaphtalin mag hierbei der Ausgangspunkt sein. Dieses Trichlornaphtalin kann dargestellt werden, nicht nur aus den Nitroverbindungen der $\beta$ - und $\gamma$-Dicblornaphtaline sondern auch aus dem $\beta$-Dinitronaphtalin. Es befinden sích also in jener Verbindung die drei Chloratome in ganz derselben gegenseitigen Stellung wie die Chloratome des $\beta$-Dichlornapthalins, des $\gamma$-Dichlornaphtalins und des von mir jetzt dargestellten $\zeta$-Dichlornaphtalins.

Das $\beta$-Dichlornaphtalin aber ist von mir aus Nitronaphtol dargestellt, welche letzte Verbindung, wie Liebermann gezeigt hat, die beiden Seitenketten in der $\alpha$-Stellung enthalten muss. Das $\gamma$-Dichlornaphtalin ist von Cleve kürzlich (Privatmittheilung) aus einer Nitrosulfonsäure dargestellt, die er sowohl aus Nitronaphtalin als aus $\alpha$-Naphtalinsulfonsäure bekommen hat und die darum unzweifelhaft nur $\alpha$-Stellungen enthält. Es sind darnach beide isomeren $\beta$ - und $\gamma$-Dichlornaphtaline als $\alpha-\alpha$-Verbindungen aufzufassen und es müssen sich in dem Naphtalinmolekül wenigstens drei $\alpha$-Stellungen vorfinden. Da aber das $\delta$-Trichlornaphtalin diesen beiden Dichlornaphtalinen ent spricht, so muss daraus gefolgert werden, das in dem $\delta$-Trichlor naphtalin allediedrei Chloratome $\alpha-S t e l l u n g e n$ besitzten.

Durch die Ueberführung des $\delta$-Trichlornaphtalins in eine Dichlorphtalsäure ist ferner in dem vorigen Aufsatz von mir der Nachweis 